\title{
Optic nerve glioma and neovascular glaucoma: report of a case
}

\author{
T. A. S. BUCHANAN AND W. F. HOYT \\ From the Neuro-Ophthalmology Unit and the Departments of Neurological Surgery, Neurology, \\ and Ophthalmology, School of Medicine, University of California San Francisco, San Francisco, \\ California 94134, USA
}

SUMMARY Neovascular glaucoma in a blind eye was a complication of an enlarging intraconal optic glioma in an 11-year-old girl. Venous stasis retinopathy was observed several months before the appearance of iris neovascularisation and elevated intraocular pressure. We suggest that venous stasis retinopathy in a patient with optic glioma is a reasonable indication for resection of the intraconal portion of the tumour.

Neovascular glaucoma is an unusual complication of optic nerve glioma. This report describes a young patient with monocular blindness from an enlarging intraconal optic glioma. After a progressive increase in proptosis she developed venous stasis retinopathy and neovascular glaucoma.

\section{Case report}

The patient, an 11-year-old girl, was first seen by her ophthalmologist in December 1978 after a school nurse discovered that she had defective vision in her left eye. Visual acuity in this eye improved from $6 / 7 \cdot 5+4$ to $6 / 6+4$ with a $-0 \cdot 75$ dioptre spherical lens. Vision in her right eye was $6 / 6+4$ unaided, but she had a $1+$ afferent pupillary defect and mild swelling of the optic disc in this eye. The left disc was normal. Colour vision, extraocular movements, and exophthalmometer readings were normal. Examination with a slit-lamp showed no abnormalities. The intraocular pressure was $15 \mathrm{mmHg}$ bilaterally. A visual field examination revealed a relative defect in the inferior nasal quadrant of the right eye. The left visual field was full.

A computerised tomography (CT) scan showed fusiform, intraconal enlargement of the right optic nerve to 2-3 times its normal diameter, the enlarged segment extending from immediately behind the globe to the orbital apex. The right optic canal was enlarged. There was no evidence of intracranial extension. The intracranial optic nerve, suprasellar

Correspondence to Professor W. F. Hoyt, c/o Department of Neurological Surgery. Editorial Office, 350 Parnassus Ave., Suite 807. San Francisco, CA 94117. USA. cistern, and ventricles were normal. Tomograms showed enlargement of the right optic canal at both its orbital and intracranial ends; its orbital diameter was $1.5-2.0 \mathrm{~mm}$ greater than that of the left canal. The walls of the canal were normal. Pneumoencephalography with polytomography confirmed that the intracranial segment of the right optic nerve was normal in diameter. A glioma was suspected. A general physical examination revealed no signs of neurofibromatosis.

Three months later the patient complained of painless dimming of vision in the right eye. Visual acuity was $6 / 9$ unaided, improving to $6 / 7 \cdot 5$ with correction; $1.5 \mathrm{~mm}$ of proptosis and a $4+$ afferent pupillary defect were noted. Swelling and congestion of the right optic disc were noticeably greater than before. Visual field loss was more marked, with an inferior altitudinal defect to small targets.

In November 1980 visual acuity in the right eye had deteriorated to the extent that she could see to count fingers at 6 inches $(15 \mathrm{~cm})$ in one quadrant only. Right proptosis had increased to $3 \mathrm{~mm}$. Intraocular pressure was $17 \mathrm{mmHg}$ bilaterally. Swelling of the right disc had increased, and there was marked venous stasis retinopathy, with optociliary shunt vessels and intraretinal haemorrhages around the optic nerve head. The left disc was normal (Fig. 1).

A month later she had 2 episodes of acute pain in her right eye, each of which resolved spontaneously. She was admitted to hospital for further examination. She was unable to perceive light and had $12 \mathrm{~mm}$ of axial, irreducible proptosis in her right eye. The eye showed marked ciliary injection. The right pupil was fixed in response to light stimulation and was widely dilated 


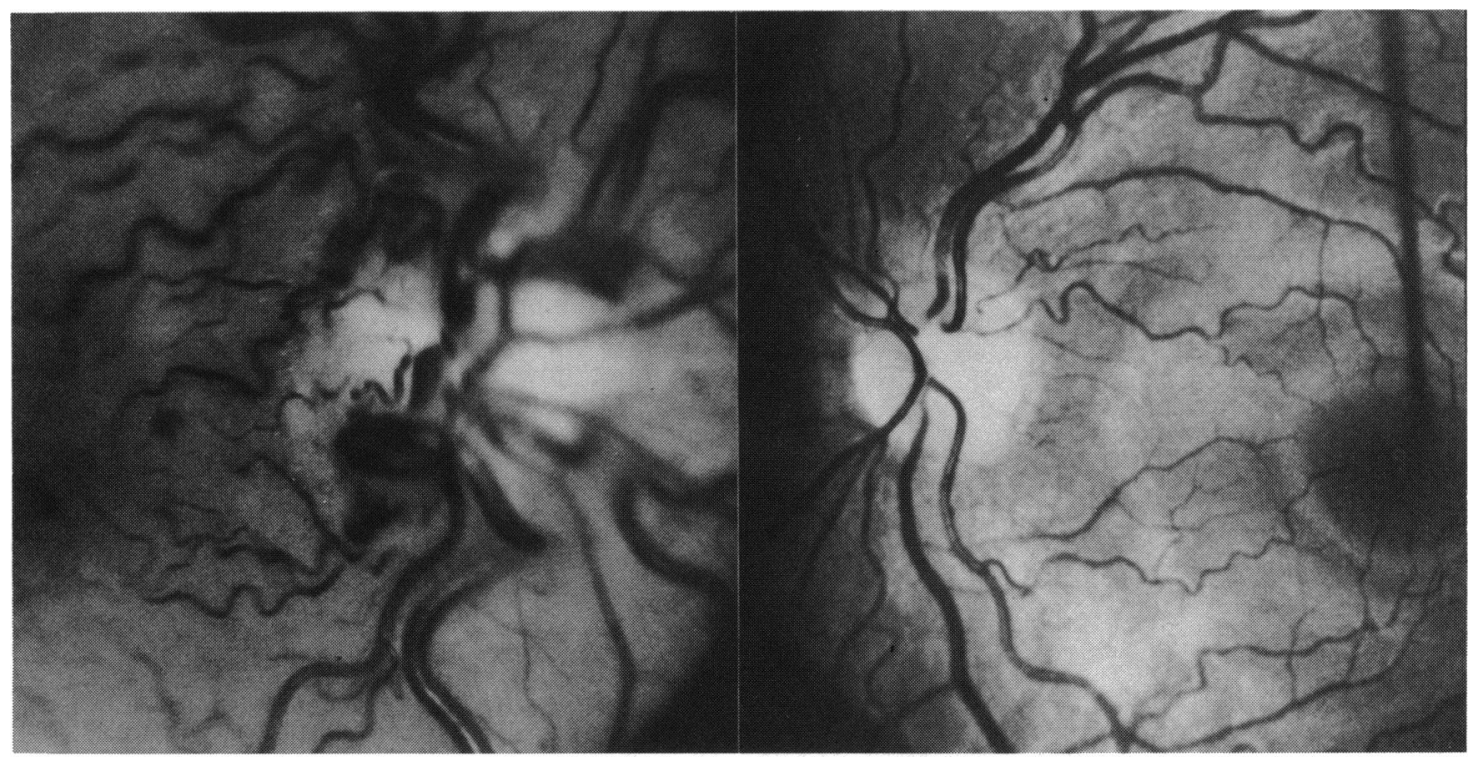

Fig. 1 Fundus photographs of both eyes. The right disc is swollen and shows marked venous stasis retinopathy, optociliary shunt vessels, and intraretinal haemorrhages around the optic nerve head. The left disc is normal.

$(10 \mathrm{~mm})$. Examination with a slit-lamp revealed corneal oedema, a shallow anterior chamber, and extensive neovascularisation and atrophy of the iris (Fig. 2). The angle of the anterior chamber was completely closed by neovascular membrane over 360 degrees. Intraocular pressure $49 \mathrm{mmHg}$. The right disc was pale and swollen and showed signs of marked arteriolar narrowing, marked venous dilatation, scattered venous haemorrhages, and optic disc atrophy. A second CT scan showed enlargement of the intraconal optic nerve mass and contrast enhancement of the periphery of the tumour (Fig. 3). The suprasellar cistern and intracranial optic nerves were normal.

Through a Kronlein orbitotomy the anterior portion of the tumour was excised from directly behind the globe and as far posteriorly as the exposure would allow. Histopathological examination showed a pilocytic astrocytoma; the central retinal vein was not occluded, and the central retinal artery was not identified. Postoperatively the intraocular pressure was $42 \mathrm{mmHg}$. The retinal arterioles were bloodless and barely visible; the retinal veins contained blood but were small. The patient's eye remains pain-free with topical medical therapy.

\section{Discussion}

Neovascular glaucoma is a rare complication of an optic nerve glioma. It has been reported in 2 cases of small gliomas, following central retinal vein occlusion in one case ${ }^{1}$ and following anterior segment ischaemia in the other. ${ }^{2}$ The initial manifestation of an optic glioma in our patient was visual loss and swelling of the optic disc. Progressive enlargement of the tumour, observed clinically and by CT, was associated with (1) increasing proptosis, (2) decreased vision, (3) progressive optic atrophy and optociliary

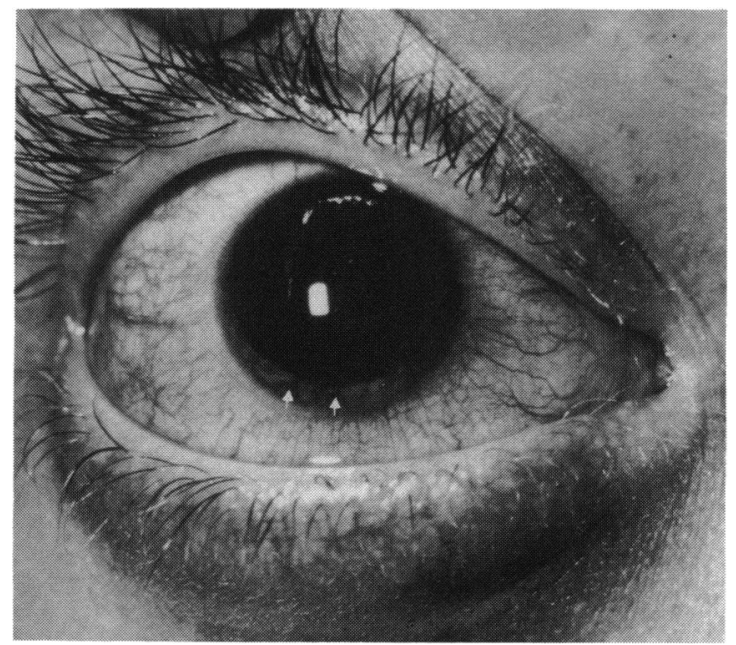

Fig. 2 Right eye showing marked ciliary injection and extensive atrophy and neovascularisation of the iris (arrows). 


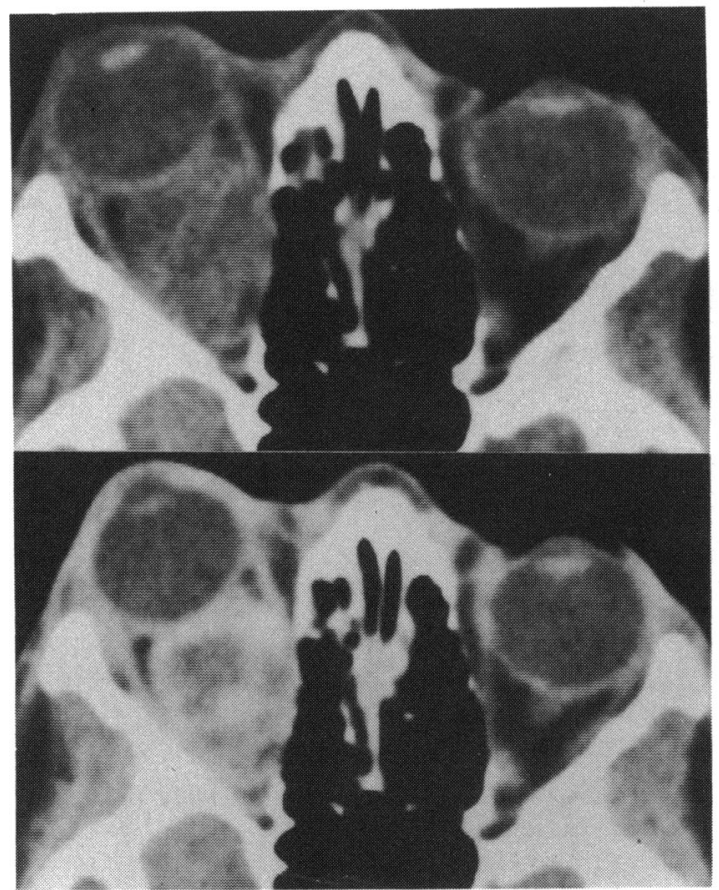

Fig. 3 Precontrast (above) and postcontrast (below) axial computerised tomography scans, the latter showing peripheral enhancement of the large optic nerve glioma.

shunts, (4) signs of venous stasis retinopathy, and (5) neovascularisation and extensive atrophy of the iris with subsequent neovascular glaucoma.

The pathogenesis of rubeosis iridis and neovascular glaucoma is not fully understood. Ashton ${ }^{3}$ proposed that ischaemic retina releases a vasoproliferative factor that diffuses into the aqueous humour, producing neovascularisation of the iris and the angle of the anterior chamber, and glaucoma.

Iris neovascularisation and neovascular glaucoma have been observed (1) following ischaemic central retinal vein occlusion, ${ }^{45}(2)$ secondary to occlusion of the central retinal artery, either alone ${ }^{67}$ or in combination with occlusion of the ciliary arteries by emboli or in temporal arteritis, ${ }^{89}$ and (3) as a complication of chronic retinal detachment. ${ }^{1011}$
We believe that iris neovascularisation and neovascular glaucoma in our patient were the result of the adverse effects of the enlarging tumour on the ciliary and retinal circulation and subsequent retinal ischaemia. Venous stasis retinopathy, marked retinal arteriolar narrowing, and extensive atrophy of the iris, together with a markedly dilated pupil, provided further evidence of retinal and ciliary ischaemia.

Preservation of the eye is a major objective in the management of orbital optic glioma. ${ }^{12}$ Our case illustrates how this goal was compromised by the development of neovascular glaucoma while the patient awaited a cosmetic operation to relieve proptosis. Although this complication is very rare, we now believe that venous stasis retinopathy in a patient with an optic glioma is a reasonable indication for resection of the intraconal portion of the tumour.

This study was supported in part by fellowship grants from the Royal College of Surgeons of England, Keeler Instruments, Ltd (UK), and the Eastern Health and Social Services Board, Northern Ireland. The authors thank Susan Eastwood for editorial assistance and Beverly J. Hunter for preparation of the manuscript.

\section{References}

1 Hovland KR, Ellis PP. Hemorrhagic glaucoma with optic nerve glioma. Arch Ophthalmol 1966; 75: 806-9.

2 Donaldson DD. Atlas of External Diseases of the Eye. Anterior Chamber, Iris, and Ciliary Body. St Louis: Mosby, 1973: 4: 238.

3 Ashton N. Retinal vascularization in health and disease. Am J Ophthalmol 1957; 44: 7-17.

4 Hayreh SS. So-called central retinal vein occlusion. Ophthalmologica 1976; 172: 1-13.

5 Laatikainen L, Blach RK. Behaviour of the iris vasculature in central retinal vein occlusion: a fluorescein angiographic study of the vascular response of the retina and the iris. Br J Ophthalmol 1977; 61: 272-7.

6 Perraut LE, Zimmermann LE. The occurrence of glaucoma following occlusion of the central retinal artery. Arch Ophthalmol 1959; 61: 845-67.

7 Wolter JR, Ryan RW. Atheromatous embolism of the central retinal artery. Arch Ophthalmol 1972; 87: 301-4.

8 Wolter JR, Philips RL. Secondary glaucoma in cranial arteritis. Am J Ophthalmol 1965; 59: 625-34.

9 Wolter JR. Double embolism of the central retinal artery and one long posterior ciliary artery followed by secondary hemorrhagic glaucoma. Am J Ophthalmol 1972; 73: 651-7.

10 Schulze RR. Rubeosis iridis. Am J Ophthalmol 1967; 63: 487-95.

11 Hoskins HD. Neovascular glaucoma. Current concepts. Trans Am Acad Ophthalmol Otolaryngol 1974; 78: 330-3.

12 Hoyt WF, Baghdassarian SB. Optic glioma of childhood: natural history and rationale for conservative management. $\mathrm{Br} J$ Ophthalmol 1969; 53: 793-8. 